\title{
REFLEXIBLE REGULAR MAPS WITH NO NON-TRIVIAL EXPONENTS FROM RESIDUAL FINITENESS
}

\author{
JOZEF ŠIRÁŇ, ĽUBICA STANEKOVÁ and MARIÁN OLEJÁR \\ Slovak University of Technology, Bratislava, Slovakia \\ e-mail:j.siran@open.ac.uk,ls@math.sk,olejar@math.sk
}

(Received 2 January 2010; accepted 24 September 2010; first published online 10 March 2011)

\begin{abstract}
Regular maps, that is, graph embeddings with the 'highest level' of orientation-preserving symmetry, can be identified with two-generator presentations of groups $G$ of the form $\left\langle x, y ; x^{m}=y^{2}=(x y)^{n}=\ldots=1\right\rangle$; the parameters $m$ and $n$ are the valence and the covalence of the map, respectively. The element $j \in Z_{m}^{*}$ is an exponent of such a map if the assignments $x \mapsto x^{j}$ and $y \mapsto y$ extend to an automorphism of $G$. The element -1 is an exponent if and only if the map is reflexible, that is, isomorphic to its mirror image. Non-trivial exponents, $j \neq \pm 1$, induce automorphisms of the underlying graph, which are not map automorphisms but can nevertheless be considered as 'external symmetries' of the map. In this paper we show with the help of residual finiteness of triangle groups that for any given $m, n \geq 3$ such that $1 / m+1 / n \leq 1 / 2$, there exist infinitely many finite, reflexible and regular maps of valence $m$ and covalence $n$ with no non-trivial exponent.
\end{abstract}

2010 Mathematics Subject Classification. 05C25.

1. Introduction and preliminaries. Reflexible regular maps are, loosely speaking, the maps carrying the 'highest level' of symmetry among all maps on orientable surfaces. Literature about such maps is abundant and in what follows we just briefly sum up the essentials, referring the reader to $[3,5,7]$ for more information.

A map is a two-cell embedded graph on a surface, which in our case is a plane or a compact, connected, orientable real two-manifold; the two cells are the faces of the map. The underlying graph of a map may be infinite in the case of an embedding in a plane, but we always assume that every vertex has finite degree and every face is bounded by a closed walk of finite length. An automorphism of a map is a permutation of its darts, that is, edges with a direction, such that the permutation induces an automorphism of the graph as well as an orientation preserving homeomorphism of the surface that preserves faces. The automorphism group $\operatorname{Aut}(M)$ of any map $M$ is semi-regular on darts of $M$. A map $M$ is said to be regular if $A u t(M)$ acts transitively, and hence, regularly on darts of $M$. Moreover, if $A u t(M)$ contains a (normal) subgroup acting regularly on the vertex set of $M$, then we speak about a regular (normal) Cayley map.

Let $M$ be a regular map on a surface carrying a given orientation, say, clockwise. Let $b$ be a fixed dart of $M$ at a vertex $v$, meaning that $b$ is directed outwards of $v$. Owing to regularity, there is exactly one element $x \in \operatorname{Aut}(M)$ sending $b$ to the clockwise next dart $b x$ pointing out of $v$. Similarly, $\operatorname{Aut}(M)$ contains exactly one element $y$ mapping $b$ onto its reverse dart. Then the composition $x y$ maps $b$ onto the dart $b x y$ that follows 
$b$ on the boundary of a face $f$ incident to $b$. The elements $x, y$ and $x y$ may thus be thought of as rotations of $M$ about the vertex $v$ by one segment, about the centre of the dart $x$ by the angle of $\pi$ and about the centre of the face $f$ by one segment.

Obviously, in our regular map $M$, all vertices have the same degree, say, $m$, and all faces are bounded by closed walks of the same length, say $n$. In $\operatorname{Aut}(M)$ we, therefore, have the relations $x^{m}=y^{2}=(x y)^{n}=1$. Cellularity of the map means that its underlying graph is connected, which is equivalent to $\operatorname{Aut}(M)$ being generated by $x$ and $y$. Invoking regularity again, the set of darts of $M$ can be identified with the group $\operatorname{Aut}(M)$ by identifying a dart $b^{\prime}$ with the unique automorphism of $M$ that takes $b$ onto $b^{\prime}$. Consequently, the map can be identified with the presentation of $\operatorname{Aut}(M)$ given by $\left\langle x, y ; x^{m}=y^{2}=(x y)^{n}=\ldots=1\right\rangle$. Conversely, any presentation of a group $G$ in terms of two generators $x$ and $y$ in the form

$$
G=\left\langle x, y ; x^{m}=y^{2}=(x y)^{n}=\ldots=1\right\rangle
$$

corresponds to a regular map $M=M(G)$, whose darts are elements of $G$ and whose edges, vertices and faces are left cosets of the subgroups $\langle y\rangle,\langle x\rangle$ and $\langle x y\rangle$, with incidence between these elements being given by non-empty intersection. In terms of identification of the map with its automorphism group it can be shown that the regular map $M$ is a (normal) Cayley map if and only if $\operatorname{Aut}(M)$ contains a (normal) subgroup $K$ such that $K \cap\langle x\rangle=\{i d\}$ and $K \cdot\langle x\rangle=\operatorname{Aut}(M)$.

This identification also allows for regarding any finite regular map of valence $m$ and covalence $n$ as a quotient of the $(2, m, n)$-triangle group $T(2, m, n)$ with presentation

$$
T(2, m, n)=\left\langle X, Y ; X^{m}=Y^{2}=(X Y)^{n}=1\right\rangle
$$

by a normal, torsion-free subgroup of finite index. In fact, by one of the basic facts in the theory of regular maps (see, for instance, [3]), there is a one-to-one correspondence between regular maps of valence $m$ and covalence $n$ on the one hand, and torsion-free, normal subgroups of $T(2, m, n)$ of finite index on the other. Note that the triangle group $T(2, m, n)$ is infinite if and only if $1 / m+1 / n \leq 1 / 2$, which we are going to assume throughout.

Let $Z_{m}^{*}$ be the multiplicative group of units of the cyclic group $Z_{m}$ and let $j \in Z_{m}^{*}$. A regular map of valence $m$ and covalence $n$, corresponding to the group $G=\left\langle x, y ; x^{m}=\right.$ $\left.y^{2}=(x y)^{n}=\ldots=1\right\rangle$, is said to have exponent $j$ if the assignment $x \mapsto x^{j}$ and $y \mapsto y$ extends to an automorphism of $G$. It is easy to verify that a product of exponents is an exponent again and therefore exponents of a regular map of valence $m$ form a subgroup of $Z_{m}^{*}$. Note that $1 \in Z_{m}^{*}$ is always an exponent. Further, -1 is an exponent if and only if the map is reflexible, that is, isomorphic to its mirror image. In the case of a reflexible regular map, $\pm 1 \in Z_{m}^{*}$ are the trivial exponents.

Exponents of maps have been introduced and extensively studied in [5], although they have earlier appeared in map-theoretic considerations in [9]. They arise naturally when considering classification of regular maps that can be built from a given underlying graph. The process can be briefly described as follows. Let $\Gamma$ be a connected graph, with all vertices of valence $m$, which underlies a regular map; a characterisation of such graphs was given in [2]. It turns out [5] that if a rotation $x$ of darts of $\Gamma$ yields a regular embedding of this graph, that is, a regular map, then for any $j \in Z_{m}^{*}, j \neq 1$, the permutation $x^{j}$ also gives rise to a regular map with the same underlying graph $\Gamma$. The two regular maps may or may not be isomorphic; and if they are, then $j$ is an exponent of the map. 
In the fundamental paper [5] the authors raised several questions regarding exponents, such as the existence of infinitely many finite regular maps of valence $m$ with the 'full' exponent group $Z_{m}^{*}$, or - at the other extreme - infinite classes of regular maps having only trivial exponents. The first question has been addressed in [8]. A partial answer to the second question was given in [1] by a lifting construction, which, however, did not preserve covalences.

The goal of this note is to present a construction, which, for any given $m, n \geq 3$ such that $1 / m+1 / n \leq 1 / 2$ yields an infinite number of pairwise non-isomorphic finite, reflexible, regular maps of valence $m$ and covalence $n$ with no non-trivial exponents. We also prove a corresponding result for reflexible regular normal Cayley maps.

2. Reflexible regular maps without non-trivial exponents. Our construction will be based on residual finiteness of triangle groups. In general, a group $H$ is said to be residually finite if for any non-identity element, $h \in H$, there exists a normal subgroup $N$ of $H$ of finite index such that $h \notin N$. Equivalently, $H$ is residually finite if for each subset $S$ of $H$ not containing the identity there exists a normal subgroup $N$ of $H$ of finite index such that $N \cap S=\emptyset$. As a consequence of a very general result of Maltsev [4] on residual finiteness of matrix groups, every triangle group $T(2, m, n)$ is residually finite. One even has a slightly stronger result.

THEOREM 1. Let $1 / m+1 / n \leq 1 / 2$. For any finite subset $S$ of a triangle group $T(2, m, n)$ not containing the identity, there exist infinitely many normal, torsion-free subgroups $N$ of $T(2, m, n)$ of finite index such that $N \cap S=\emptyset$.

Proof. Let $1 / m+1 / n \leq 1 / 2$ and let $T(2, m, n)=\left\langle X, Y ; X^{m}=Y^{2}=(X Y)^{n}=1\right\rangle$. Let $S$ be any finite subset of $T(2, m, n)$ not containing the identity. Consider the set $S^{\prime}=S \cup\left\{X^{i} ; 1 \leq i \leq m-1\right\} \cup\{Y\} \cup\left\{(X Y)^{i} ; 1 \leq i \leq n-1\right\}$. By residual finiteness, $T(2, m, n)$ contains a normal subgroup $N$ of finite index, avoiding $S^{\prime}$. Suppose $N$ contains an element of finite order. By normality and by the well-known fact that every element of $T(2, m, n)$ of finite order is conjugate to the power of $X, Y$ or $X Y$, we conclude that $N$ would have to contain an element of $S^{\prime}$, which is a contradiction. This shows that $N$ is torsion-free.

We can now take any non-identity element of $N$ and include it in the set $S^{\prime}$. The above procedure yields a normal, torsion-free subgroup of finite index in $T(2, m, n)$, which is different from $N$ and still avoids $S$. Our theorem now follows by induction.

We will use this fact in the proof of our main result.

THEOREM 2. For any $m, n \geq 3$ such that $1 / m+1 / n \leq 1 / 2$ there exist infinitely many pairwise non-isomorphic reflexible regular maps of valence $m$ and covalence $n$ with no non-trivial exponents.

Proof. Consider the triangle group $H=T(2, m, n)$ for fixed $m, n$ as in the statement of our theorem; the condition $1 / m+1 / n \leq 1 / 2$ implies that $H$ is infinite. Let $H$ have presentation as in equation (2), that is,

$$
H=\left\langle X, Y ; X^{m}=Y^{2}=(X Y)^{n}=1\right\rangle,
$$

and let $M(H)$ be the regular map corresponding to this presentation. It is well known [3] that $M(H)$ is a tessellation $U(m, n)$ of an infinite plane with congruent $n$-gons, $m$ of which meet at every vertex. Here the adjective 'congruent' refers to the Euclidean or 
hyperbolic geometry, depending on whether $1 / m+1 / n$ is equal to or smaller than $1 / 2$, respectively. In the corresponding geometry, $X, Y$ and $X Y$ are rotations of the plane by the angle $2 \pi / m, \pi$ and $2 \pi / n$ about a fixed vertex, the centre of a fixed edge incident to $v$ and about the centre of a fixed face incident to the edge, respectively, preserving the tessellation $U(m, n)$.

The assignment $X \mapsto X^{-1}$ and $Y \mapsto Y$ extends to an automorphism $\sigma$ of $H$. In order to see this it is sufficient to realise that this assignment preserves the defining relations of $H$ since the order of $X^{-1} Y=X^{-1} Y^{-1}=(Y X)^{-1}$ is equal to the order of $X Y$. It follows that -1 is an exponent of $M(H)$, which implies the known fact that the map $M(H)$ is reflexible.

If $1 / m+1 / n \leq 1 / 2$, the identification of the triangle group $H$ with the infinite plane tessellations $M(H)=U(m, n)$ implies the important fact that for any $j \in Z_{M}^{*}$ such that $j \notin\{1,-1\}$, the element $X^{j} Y$ has infinite order in $H$.

Let $S=\left\{\left(X^{j} Y\right)^{n} ; j \in Z_{m}^{*}, j \neq \pm 1\right\}$. By Theorem 1, the group $H=T(2, m, n)$ contains an infinite number of normal, torsion-free subgroups of finite index, all avoiding the set $S$. Let $N^{\prime}$ be any such subgroup. Invoking the automorphism $\sigma$ of $H$ considered above, let $N=N^{\prime} \cap \sigma\left(N^{\prime}\right)$. Clearly, $N$ is also a torsion-free, normal subgroup of finite index in $H$ that avoids $S$; moreover, note that $\sigma(N)=N$. We show that the regular map $M$ corresponding to the quotient group $H / N$ does not contain any exponent $j \in Z_{m}^{*}$ except $j= \pm 1$.

Indeed, assume that some $j \in Z_{m}^{*}$ is an exponent of $M$. Identifying $M$ with the group $H / N$ that has a partial presentation of the form $\left\langle x, y ; x^{m}=y^{2}=(x y)^{n}=\ldots=\right.$ 1), where $x=N X$ and $y=N Y$, this means that $H / N$ has an automorphism that takes $x$ onto $x^{j}$ and fixes $y$. It follows that the elements $x y$ and $x^{j} y$ must have the same order in $H / N$. In particular, $\left(x^{j} y\right)^{n}=1$ in $H / N$, that is, $\left(X^{j} Y\right)^{n} \in N$. But if $j \neq \pm 1$, this is a contradiction with the fact that $N \cap S=\emptyset$. On the other hand, if $j=-1$, then the automorphism $\sigma$ of $H$ projects onto an automorphism of $H / N$ that inverts $x$ and fixes $y$ since $\sigma(N)=N$. This shows that $M$ is reflexible.

Obviously, the infinitude of the subgroups $N^{\prime}$ implies infinitude of the subgroups $N$. By the theory of regular maps [3], a pair of non-isomorphic torsion-free, normal subgroups $N$ of finite index in $H=T(2, m, n)$ give rise to non-isomorphic, finite, regular maps of vertex valence $m$ and face length $n$. This completes the proof.

The regular Cayley maps have been extensively studied in [6], where it has been shown that if such a map has valence $m$ and covalence $n$, then either $m$ is a divisor of $n$ or $m / 2$ is odd and divides $n$. We now show that for $m$ and $n$ satisfying this condition the previous result can be extended to regular normal Cayley maps.

THEOREM 3. Let $m, n \geq 3$ be integers, both not equal to 3 , such that either $m$ divides $n$ or $m / 2$ is an odd integer dividing $n$. Then, there exist infinitely many pairwise nonisomorphic reflexible regular normal Cayley maps of valence $m$ and covalence $n$ with no non-trivial exponents.

Proof. We will use the notation introduced in the previous proof. The conditions on $m, n$ imply that $1 / m+1 / n \leq 1 / 2$ and so the triangle group $H=\left\langle X, Y ; X^{m}=Y^{2}=\right.$ $\left.(X Y)^{n}=1\right\rangle$ is infinite. Further, the conditions on $m, n$ guarantee the existence of the group epimorphism $\theta: H \rightarrow Z_{m}=\{0,1, \ldots, m-1\}$ given by $\theta(X)=1, \theta(Y)=0$ if $m$ divides $n$, and $\theta(X)=1, \theta(Y)=m / 2$ if $m / 2$ is an odd divisor of $n$. For the normal subgroup $K=\operatorname{ker}(\theta)$ of $H$, we have $K \cap\langle X\rangle=\{i d\}$ and $K \cdot\langle X\rangle=H$, that is, the tessellation $U(m, n)=M(H)$ is a regular normal Cayley map. 
Recall now the isomorphism $\sigma$ of $H$ inverting $X$ and preserving $Y$ that establishes reflexibility of the tessellation $U(m, n)=M(H)$. Since the image of the epimorphism $\theta$ : $H \rightarrow Z_{m}$ is an Abelian group, one can verify that $\theta(W)=0$ if and only if $\theta(\sigma(W))=0$. This means that the kernel $K$ of $\theta$ satisfies $\sigma(K)=K$.

Let $N$ be any of the (infinitely many) normal, torsion-free subgroups of $H$ of finite index constructed in the proof of Theorem 2, with $\sigma(N)=N$. The group $L=K \cap N$ is obviously a normal, torsion-free subgroup of $H$ of finite index, such that $\sigma(L)=L$. Since $L$ is a subgroup of $N$, by the previous proof $L$ does not contain any element from the set $S=\left\{\left(X^{j} Y\right)^{n} ; j \in Z_{m}^{*}, j \neq \pm 1\right\}$. Hence, the reflexible regular map corresponding to the quotient group $H / L$ does not contain any exponent $j \in Z_{m}^{*}$ except, of course, $j= \pm 1$. Moreover, this map is also a normal Cayley map for the group $K / L$, because $K / L$ is a normal subgroup of $H / L$ such that $H / L=K / L \cdot\langle X L\rangle$ and the intersection $(K / L) \cap\langle X L\rangle$ is trivial. Our result follows.

It remains to compare our findings with the earlier results of [1] on this topic. In [1] it was proved that for any $m \geq 3$ there exist infinitely many finite, chiral (that is, non-reflexible) regular maps of valence $m$ with no exponent except 1 . The face lengths of these maps, however, are multiples of sufficiently large primes and hence not under control. Our present result, in contrast, yields infinitely many finite, regular maps of prescribed valence $m$ and prescribed face length $n$ (where $1 / m+1 / n \leq 1 / 2$ ) containing no exponents except \pm 1 . Thus, allowing -1 as an exponent, that is, allowing reflexibility, we have both the vertex valence and the face length under control and still can exclude all non-trivial exponents. In addition, for 'admissible' vertex valences and face lengths, we have a stronger result for reflexible regular normal Cayley maps.

ACKNOWLEDGEMENTS. This research was supported by the VEGA Research Grant No. 1/0489/08, the APVV Research Grant Nos. 0040-06 and 0104-07 and the APVV LPP Research Grant Nos. 0145-06 and 0203-06.

\section{REFERENCES}

1. D. Archdeacon, P. Gvozdjak and J. Širáň, Constructing and forbidding automorphisms in lifted maps, Mainath. Slovaca 47 (1997), 113-129.

2. A. Gardiner, R. Nedela, M. Škoviera and J. Širán̆, Characterization of graphs which underlie regular maps on closed surfaces, J. Lond. Math. Soc. (2) 59(1), (1999), 100-108.

3. G. A. Jones and D. Singerman, Theory of maps on orientable surfaces, Proc. Lond. Math. Soc. 37(3) (1978), 273-307.

4. I. Maltsev, On the faithful representation of infinite groups by matrices, Mat. Sb. 8(50) (1940) 405-422 (Russian), Amer. Math. Soc. Transl. 45(2) (1965), 1-18 (English).

5. R. Nedela and M. Škoviera, Exponents of orientable maps, Proc. Lond. Math. Soc. 75(3) (1997), 1-31.

6. R. B. Richter, J. Širáň, R. Jajcay, T. W. Tucker and M. E. Watkins, Cayley maps, J. Comb. Theory Ser. B 95 (2005), 189-245.

7. J. Šráń, Regular maps on a given surface: A survey, in Topics in discrete mathematics (R. Thomas et al., Editors), Springer series no. 26: Algorithms and combinatoric (Springer, New Mexico, 2006), 591-609.

8. J. Širán̆ and Y. Wang, Maps with highest level of symmetry that are even more symmetric than other such maps: Regular maps with largest exponent groups, Contemporary Math. (AMS series) 531 (2010), 95-102.

9. S. E. Wilson, Operators over regular maps, Pacific J. Math. 81 (1979), 559-568. 